\title{
A Systems Approach to the Cellular Analysis of Associative Learning in the Pond Snail Lymnaea
}

\author{
Paul R. Benjamin, ${ }^{1}$ Kevin Staras, and György Kemenes \\ Sussex Centre for Neuroscience, School of Biological Sciences, University of Sussex, Falmer, Brighton, East Sussex, BN1 9QG, UK
}

\begin{abstract}
We show that appetitive and aversive conditioning can be analyzed at the cellular level in the well-described neural circuitries underlying rhythmic feeding and respiration in the pond snail, Lymnaea stagnalis. To relate electrical changes directly to behavior, the snails were first trained and the neural changes recorded at multiple sites in reduced preparations made from the same animals. Changes in neural activity following conditioning could be recorded at the level of motoneurons, central pattern generator interneurons and modulatory neurons. Of significant interest was recent work showing that neural correlates of long-term memory could be recorded in the feeding network following single-trial appetitive chemical conditioning. Available information on the synaptic connectivity and transmitter content of identified neurons within the Lymnaea circuits will allow further work on the synaptic and molecular mechanisms of learning and memory.
\end{abstract}

A large body of work using the very successful cellular and molecular approach to learning and memory has provided important models of plasticity in both invertebrates and vertebrates (Byrne 1987; Milner et al. 1998). These studies emphasize changes occurring at a single locus. However, there is increasing evidence to suggest that most forms of learning involve changes at several different sites in the brain (Wolpaw 1997; Lisberger 1998), suggesting that more complex models will be required. The identification of multiple sites of plasticity requires a systems approach to the analysis of learning and memory, which makes it important to first identify the electrical changes that result from conditioning throughout a neural network and then attempt to relate these to behavioral plasticity. Given the complexity of the brain, systems-level analyses of learning and memory have often been inevitable in vertebrate studies striving to link learning-related behavioral and cellular changes. In our opinion there is a clear requirement for a similar approach in numerically more simple invertebrate systems, where 100-1000s of neurons form circuits, especially because of the evidence that the invertebrate central nervous system (CNS) can also show changes resulting from conditioning in several different structures (e.g., antennal lobes, mushroom bodies, and lateral protocerebral lobes of the honeybee; Hammer 1997). A systems approach to invertebrate learning is particularly useful if we have no prior information about the numbers and locations of sites of plasticity in the various elements of the nervous system. Despite the recent developments in in vivo single-cell recording and imaging techniques in vertebrates (Fetcho and O'Malley 1997; Svoboda et al. 1997), studies using invertebrates still have the added advantage that more specific learning-related

${ }^{1}$ Corresponding author.

E-MAIL P.R.Benjamin@sussex.ac.uk; FAX 441273678535. changes in electrical activity at the single identified cell or single identified synapse level can be examined throughout a specific behavioral neural network.

One of the most promising candidates for a systemslevel analysis of learning in invertebrates is the pond snail Lymnaea stagnalis where a number of behavioral associative conditioning paradigms have been used successfully and, importantly, cellular traces of behavioral conditioning have been found in both isolated and semi-intact preparations. The discovery that the feeding response of Lymnaea can be subjected to both appetitive and aversive classical conditioning (Audesirk et al. 1982; Kemenes and Benjamin 1989a; Kojima et al. 1996), together with the fact that the circuit-generating feeding ingestion movements is well known (Benjamin and Elliott 1989; Yeoman et al. 1995; Brierley et al. 1997; Staras et al. 1998a), has made feasible a cellular analysis of feeding-related associative memory formation. Aversive operant conditioning of the respiratory behavior also has been demonstrated in Lymnaea (Lukowiak et al. 1996, 1998) and because the circuit underlying respiration is another network that has been described in detail (Syed et al. 1990; Syed and Winlow 1991b), it was also possible to subject this form of learning to a cellular analysis (Spencer et al. 1999).

To understand the learning-induced electrical changes in the neural networks underlying feeding and respiration it is necessary first to consider their organization at the cellular level (Fig. 1).

\section{The Feeding Network}

Feeding in Lymnaea consists of a sequence of repetitive movements called rasps. During each rasp, the mouth opens and a toothed radula is scraped forward over the food substrate (protraction phase). Food is then lifted into the

LEARNING \& MEMORY 7:124-131 @ 2000 by Cold Spring Harbor Laboratory Press ISSN1072-0502/00 \$5.00

$$
\begin{array}{lllllllllllllll}
L & E & A & R & N & I & N & G & \mathbf{Q} & M & E & M & O & R & Y \\
\text { www.learnmem.org } & & & &
\end{array}
$$




\section{A Feeding network}

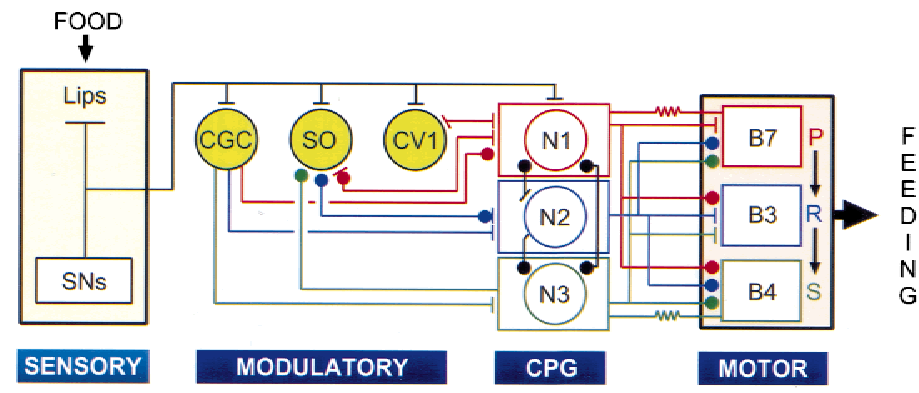

B Respiratory network

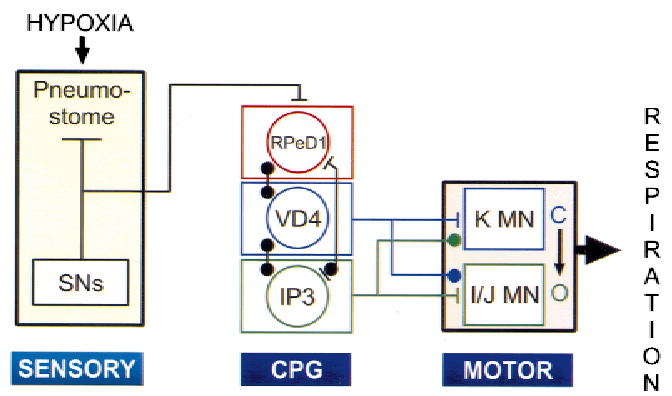

Figure 1 The feeding and respiratory networks of Lymnaea. (A) The neuronal network underlying rhythmic feeding behavior. Chemosensory neurons (SNs) located in the lip structures detect the presence of food or chemostimulants, such as sucrose. The sensory pathway is not known in detail but excitatory inputs are distributed in parallel to modulatory neurons (solid yellow-green circles), including the cerebral giant cells (CGCs), the slow oscillator (SO), and the cerebral ventral 1 (CV1) and the N1 CPG interneurons (red circle). The complex synaptic connectivity between these cells leads to activation of the CPG, with the protraction (N1), rasp (N2, blue circle), and swallow (N3, green circle) phases of the feeding cycle following in sequence because of the synaptic connectivity within the CPG and their intrinsic properties. Several subtypes of each of the $\mathrm{N}$ cells have been characterized but because of the complexity of their synaptic connections with modulatory interneurons and motoneurons, only a generalized representative of each $\mathrm{N}$ type is shown. The rhythmic pattern generated by the CPG drives protraction ( $P$, red square), rasp ( $R$, blue square), and swallow ( $S$, green square) phase motoneurons, such as B7, B3, and B4, leading to sequences of muscular activity and feeding movements. (B) The neuronal network underlying respiratory behavior. Activation of sensory neurons (SNs) located in the pneumostome-osphradial area leads to activation of the respiratory CPG, comprised of RPeD1 (red circle), VD4 (blue circle), and IP3 (green circle). VD4 drives activity in K MN (motoneurons, blue square), responsible for pneumostome closing (inspiration), and IP3 drives activity in I/J MN (green square), responsible for pneumostome opening (expiration). (Solid circles) Chemical inhibitory synapses; (vertical bars) chemical excitatory synapses; (horizontal bar) peripheral sensory endings; (Crooked lines) electrotonic connections.

mouth (retraction phase), which closes while the food is being swallowed (swallow phase) and the sequence is repeated (Rose and Benjamin 1979). Rhythmic movements of the feeding muscles are driven by a network of motoneurons (B1-B10) that, in turn, are driven by synaptic inputs from a feeding central pattern generator (CPG) network of interneurons (Benjamin and Rose 1979). Each phase of the feeding rhythm is generated by one of three main types of CPG interneurons, N1 (protraction), N2 (retraction), or N3 (swallow), providing sequences of excitatory and inhibitory synaptic inputs to motoneurons active in different phases of the feeding rhythm (Rose and Benjamin 1981; Elliott and Benjamin 1985a; Yeoman et al. 1995; Brierley et al. 1997). CPG-driven rhythmic electrical activity can be recorded in the feeding network even in the absence of feeding muscles and this is called fictive feeding. Activity in the motoneurons and CPG neurons is modulated by identified higherorder interneurons, such as the cerebral giant cells (CGCs, McCrohan and Benjamin 1980; Yeoman et al. 1996), cerebral ventral 1 cells (CV1s, McCrohan 1984), and the slow oscillator neuron (SO, Elliott and Benjamin 1985b). The components of the feeding network and their synaptic connectivity have thus been extensively characterized and are summarized in Figure 1A.

Sucrose is an effective chemical stimulus for feeding (Kemenes et al. 1986; Staras et al. 1998b) and is therefore used as the unconditioned stimulus (US) in appetitive classical conditioning. Interestingly, the same sucrose stimulus can serve as a conditioned stimulus (CS) in an aversive clas- sical conditioning paradigm if it is consistently paired with aversive unconditioned stimuli (Kojima et al. 1996). At the cellular level, sucrose applied to the lips in semi-intact preparations induces fictive feeding in motoneurons and interneurons of the feeding network. As well as the CPG neurons, the modulatory CGC and CV1 cells are also activated in the sucrose-driven fictive feeding rhythm (Kemenes et al. 1986; Whelan and McCrohan 1996) and so are likely to play an important role in pattern generation via their synaptic connections with the CPG (Fig. 1A). Aversive chemical stimuli (e.g., quinine), which inhibit feeding in intact animals, were shown to inhibit ongoing fictive feeding recorded on both modulatory neurons and motoneurons of the feeding network (Kemenes et al. 1986).

The CS for appetitive classical conditioning in Lymnaea is either a chemical (amyl acetate) or a tactile stimulus (a gentle brush stroke applied to the lips). Neither conditioned stimuli will evoke feeding before training but CS-US pairing(s) results in conditioned feeding responses (Audesirk et al. 1982; Kemenes and Benjamin 1989a). The CS used in most of the appetitive learning experiments where electrophysiological work was carried out is a tactile stimulus applied to the lips. The effects of the CS on feeding neurons in naive snails were recently examined in detail (Staras et al. 1999b) to provide important background information for the learning studies. It confirmed previous results (Kemenes et al. 1986) that touch to the lips, monitored either at the behavioral or the electrophysiological level, cannot initiate or maintain feeding but showed that a single touch

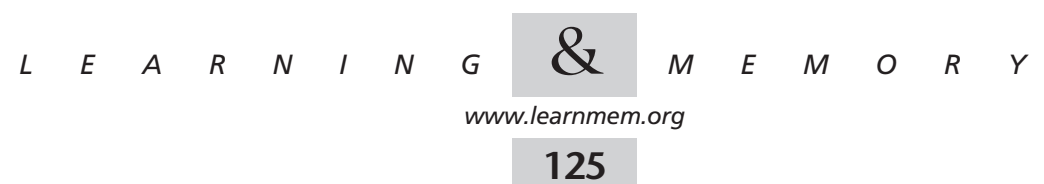


to the lips produced a complex sequence of inhibitory and excitatory synaptic inputs on all neurons of the feeding network.

\section{The Respiratory Network}

The aquatic pulmonate snail Lymnaea can breath either through the skin or through a simple lung. As their name implies, L. stagnalis populations often live in stagnant water and when the environment becomes hypoxic the snails float to the surface and perform rhythmic opening and closing movements of their pulmonary opening, the pneumostome (Syed et al. 1991). This aerial respiration behavior is controlled by a respiratory CPG (Syed and Winlow 1991b; Syed et al. 1991), the three main components of which are the right pedal dorsal 1 (RPeD1), input 3 (IP3), and visceral dorsal 4 (VD4) interneurons. These provide synaptic inputs to identified motoneurons (I and J, opener and $\mathrm{K}$, closer) innervating pneumostome opener and closer muscles (Benjamin and Winlow 1981; Syed and Winlow 1991a; Syed et al. 1991). A summary diagram of the cellular organization of the aerial respiratory network is shown in Figure 1B. The chemosensory stimulus (hypoxia) that triggers pneumostome opening first activates sensory cells in the pneumostome-osphradial area, which in turn provide excitatory afferent inputs to RPeD1 (T. Inoue, Z. Haque, M. Takasaki, K. Lukowiak, and N. Syed, unpubl.). Through its synaptic connections with the other members of the CPG network, activation of RPeD1 initiates CPG activity, which underlies the respiratory rhythm. Tactile stimulation of the pneumostome area evokes pneumostome closure and stops aerial respiratory behavior. This response, which is mediated by an identified whole-body withdrawal neuron (RPeD11, syed et al. 1991; Inoue et al. 1996a,b), is an important component of the withdrawal response in Lymnaea and therefore tactile stimulation of the pneumostome could be used as an aversive stimulus in operant conditioning experiments.

\section{Appetitive Classical Conditioning to a Tactile CS}

In the most thoroughly investigated classical conditioning paradigm used in Lymnaea, a tactile conditioning stimulus to the lips is paired with the unconditioned stimulus, sucrose (Kemenes and Benjamin 1989a,b, 1994; Staras et al. 1998b, 1999a). These experiments demonstrated that Lymnaea can be classically conditioned by repeatedly pairing touch to the lip with food (5-15 trials) and that appetitive learning in Lymnaea shares important characteristics with associative conditioning in vertebrates, such as stimulus generalization and discriminative learning (Kemenes and Benjamin 1989a), classical-operant interactions (Kemenes and Benjamin 1989b), and strong dependence on both external and internal background variables (Kemenes and Benjamin 1994).

Two approaches have been used to investigate the cel- lular basis of tactile appetitive classical conditioning in Lymnaea. One approach was based on the development of an in vitro preparation where electrophysiological manipulation of neuronal pathways aims to mimic the behavioral conditioning paradigm (Kemenes et al. 1997). In this study a lip touch stimulus was paired with intracellular activation of the modulatory SO neuron, which can drive fictive feeding. After 6-10 pairings, presentation of the touch stimulus could activate a robust fictive feeding rhythm. Given that neurons can be continuously monitored throughout the acquisition of the learned response, this approach is likely to offer a good opportunity to investigate the processes leading to memory formation. However, this is only an in vitro analog of classical conditioning that may not directly correlate with behavioral plasticity. Therefore, the second and main approach has been to use behavioral conditioning followed by electrophysiological analysis to record changes in electrical activity that follow long-term memory formation. In the semi-intact preparation, we electrophysiologically recorded fictive feeding patterns as a systems-level correlate of the repetitive feeding behavior seen in the intact snail. We have routinely used a retraction-phase feeding motoneuron, known as $\mathrm{B} 3$, as a monitor of fictive feeding in the whole network (see Fig. 1A). This cell is easy to identify, plays no role in pattern generation, but receives synaptic inputs from all three types of CPG interneurons and so can be used as an indirect monitor of the conditioned activation of the CPG. It also receives an easy-to-identify early compound excitatory postsynaptic potentials (EPSP) input that results from stimulation of the CS pathway (Staras et al. 1999b). Other motoneurons have also been recorded to check that all the feeding motoneurons are activated during conditioned fictive feeding.

Using the lip-touch training protocol (Kemenes and Benjamin 1989a), we subjected snails to 15 training trials over 3 days and then dissected these and control animals for electrophysiological analysis, starting on the day after the last training trial. Touching the lips of the intact snails from the experimental group after training induced a pattern of feeding movements not seen in controls (Fig. 2Aii) and this was significant at the statistical level (Fig. 2Ai). Similar significant differences (Fig. 2Bi) were seen between experimental and control animals at the level of the electrophysiologically recorded fictive feeding pattern in $\mathrm{B} 3$ in the semiintact preparations made from the same snails (Fig. 2Bii). The only difference in the data was that the absolute level of the rate of conditioned fictive feeding was low compared with behavioral conditioned feeding rates (cf. Fig. 2, Ai and $\mathrm{Bi}$ ). Because the CPG-driven activity in the $\mathrm{B} 3$ motoneuron depends ultimately on activity of neurons at all levels of the feeding network, we believe that the conditioned fictive feeding recorded in this cell is a systems-level readout of the memory trace in the whole feeding system. However, more detailed changes can also be recorded in different parts of

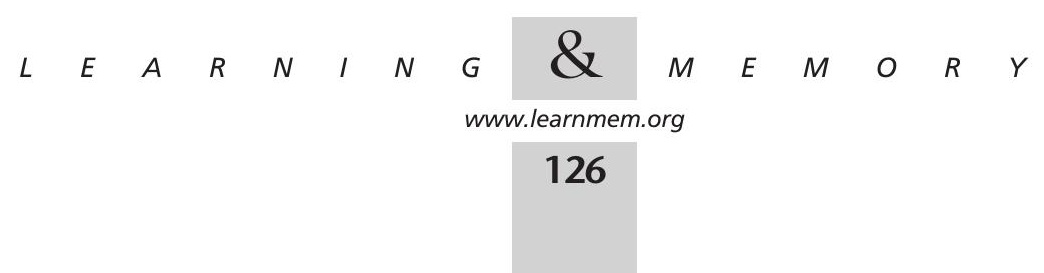



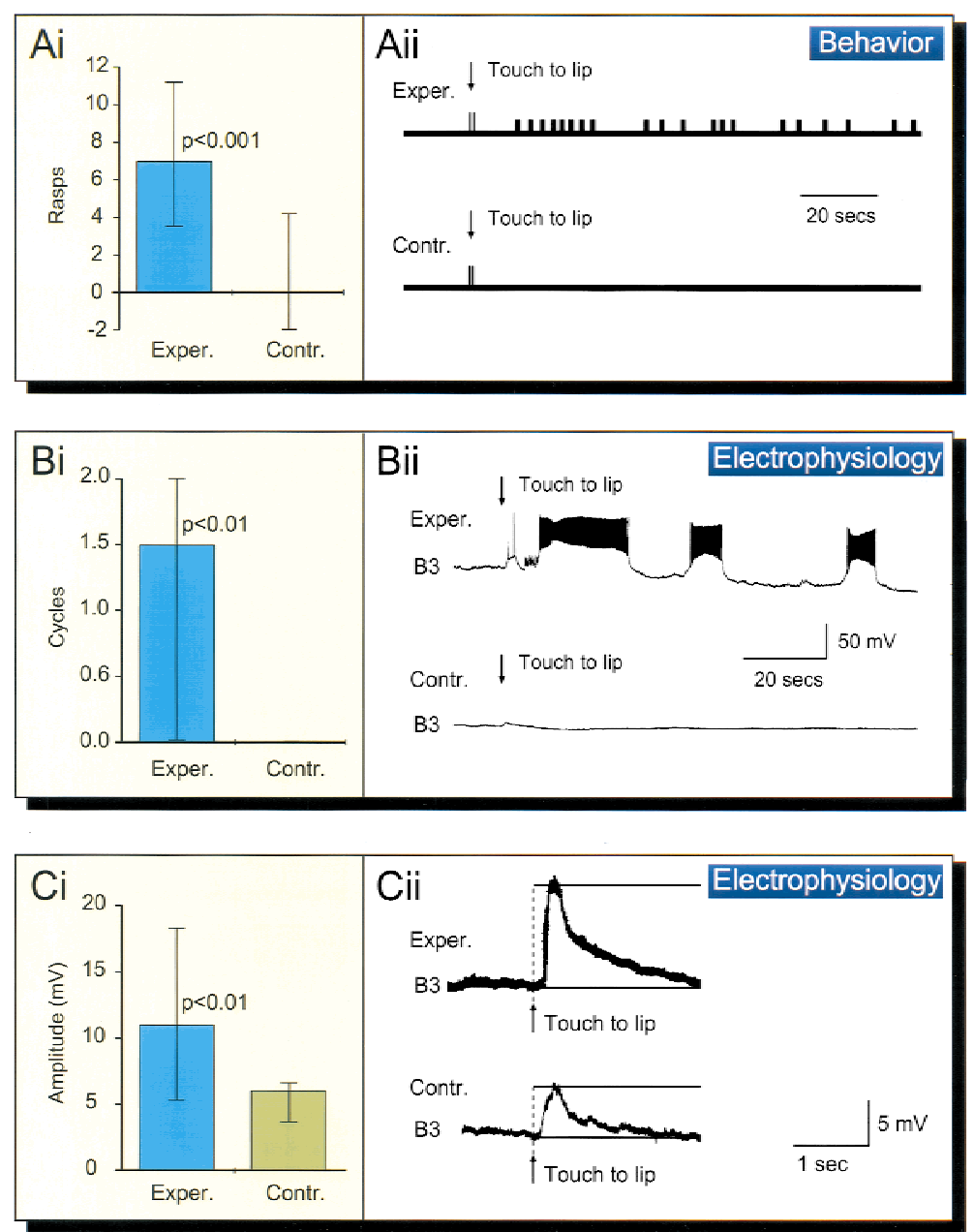

Figure 2 Cellular effects of appetitive conditioning with a tactile stimulus (touch to the lip). (A) Behaviorally-recorded feeding responses. (Ai), Change in feeding rate after tactile conditioning is significantly greater in the experimental group (15 pairings of lip tactile CS and sucrose US) vs. the control group (random CS and US presentation). (Aii) Example of feeding response to lip tactile stimulation in an experimental vs. a control animal. Solid vertical bars represent single rasp events. (B) Electrophysiologically recorded fictive feeding responses in reduced preparations derived from the behaviorally-trained animals in $A$. (Bi) Change in fictive feeding rate after tactile conditioning is significantly greater in the experimental group versus the control group. (Bii) Example of fictive feeding response to lip tactile stimulation in an experimental vs. a control semi-intact preparation monitored using the motoneuron, B3. Fictive feeding cycles are seen as characteristic inputs on the B3. (C) A cellular correlate of tactile conditioning. (Ci) Amplitude of the B3 EPSP evoked by the tactile stimulus is significantly larger in conditioned vs. control preparations. (Cii) Example of the touch-evoked EPSP in an experimental and control preparation. Statistical data are presented as medians and interquartile ranges. After Staras et al. (1999a).

the network. One of these is the early EPSP that occurs in the $\mathrm{B} 3$ before the onset of the fictive feeding pattern. The amplitude (Fig. 2C), but not the latency and duration, of the EPSP was enhanced significantly after conditioning. In sated snails the conditioned fictive feeding response to touch was lost but the increase in the EPSP amplitude persisted (Staras et al. 1999b). This suggests that there is unlikely to be a causal link between increases in amplitude in $\mathrm{B} 3$ and generation of the fictive feeding pattern.

One candidate for initiating CPG activity following conditioning is the modulatory interneuron cell type known as CV1. This neuron is capable of driving a fictive feeding neuron via its connections with the N1M cells of the CPG network and activity in these cells normally accompanies unconditioned feeding patterns stimulated by sucrose (Whelan and McCrohan 1996). Significantly, CV1 is the only protraction phase interneuron type that is depolarized by touch applied to the lips in naive snails (Staras et al. 1999b). Despite their small size, recently in ongoing studies we have been able to record a sufficient number of CV1 cells in semi-intact preparations for quantitative analysis. We find that they are significantly more active following touch in conditioned snails compared with controls and that they show the typical patterned activity seen with sugar (P.R. Benjamin et al., unpubl.)

Electrical correlates of classical conditioning were recorded at several levels within the feeding circuit and these could all be potential sites of plasticity. That some sites could be quite early in the CS pathway was revealed by extracellularly recording mechanosensory fibers located in the connective between the cerebral and buccal ganglia (Staras et al. 1999a). Tactile responses could be recorded in these fibers and, following conditioning, the number of spikes occurring early in this response increased, compared with controls.

\section{Appetitive Classical Conditioning to a Chemical CS}

In the original successful formulation of appetitive classical conditioning in Lymnaea, snails were subjected to a chemical conditioning protocol using amyl acetate as the CS and sucrose as the US (Audesirk et al. 1982). Following training, the explicitly paired (CS-US) experimental group showed significantly greater feeding responses to amyl acetate over their own naive responses and all the standard control groups (Random, Explicitly Unpaired, CS alone, US alone). As might be predicted for appetitive classical conditioning, both age and motivational state (hunger vs. satiety) influenced learning (Audesirk et al. 1982). Both hungry and sated young snails could acquire the conditioned response but in the latter group its expression was only apparent when the animals were starved before testing. On the other hand, old snails could only acquire the condi-

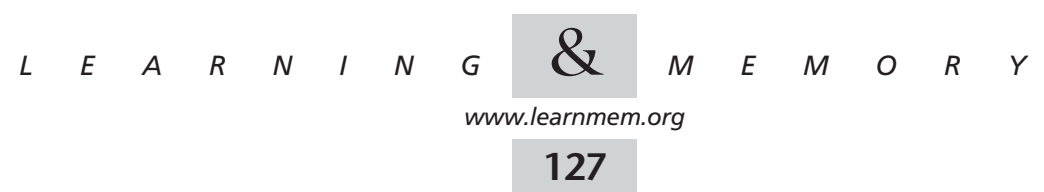


tioned response if they were maintained in a hungry state during training. The significance of motivational state became even more apparent when it was realized that if snails were starved long enough (for 5 days) before and throughout the experiment, even a single pairing of amyl acetate and sucrose resulted in long-term memory that lasted for at least 19 days (Alexander et al. 1984). This is a remarkable example of single trial learning that we have replicated in our laboratory and use now as a starting point for an analysis of the time course of the mechanisms underlying long term memory formation.

An in vitro correlate of an appetitive conditioned response was also found after chemical appetitive classical conditioning of intact animals with 15 paired applications of amyl acetate as the CS and sucrose as the US (Whelan and McCrohan 1996). The electrophysiological tests in this experiment concentrated on the CV1 cells, a pair of modulatory interneurones in the cerebral ganglia (McCrohan 1984). In most semi-intact preparations ( $80 \%)$ taken from experimental snails, application of amyl acetate to the lips led to depolarization of the membrane and in $~ 30 \%$ of them to strong rhythmical bursting activity. Interestingly, in some preparations where the buccal feeding central pattern generator system was removed, CV1 continued to show an excitatory response to amyl acetate. This indicates that, at least in the case of chemical appetitive classical conditioning, one site of plasticity may be located in the cerebral ganglia.

We have recently obtained similar results following single trial chemical classical conditioning to the same CS. The example shown in Figure 3 shows that amyl acetate can activate a fictive feeding pattern in conditioned (Fig. 3A) but not control (Fig. 3B) snails and further statistical analysis (not shown) confirmed that this difference between experimentals and controls was significant. Fictive feeding was also monitored on the $\mathrm{B} 3$ motoneuron, as in the tactile conditioning experiments, and patterned fictive feeding activity could be recorded on both CV1 and the B3. This data on chemical conditioning also indicates that training can lead to equivalent systems level responses that were previously reported for tactile conditioning except that it occurs after only 1 training trial rather than 15 . This success in getting a robust electrical correlate of chemical conditioning makes further cellular analysis possible.

\section{Aversive Classical Conditioning to a Chemical CS}

Aversive classical conditioning in Lymnaea was based on pairing sucrose with an aversive stimulus such as $\mathrm{KCl}$, which inhibits feeding and evokes a withdrawal response
(Kojima et al. 1996, 1997). After eight trials, trained animals showed a significantly weaker feeding response to sucrose than did controls and this memory lasted for more than one month (Kojima et al. 1997).

A cellular analysis of this learned response was carried out on isolated brains dissected from conditioned and control animals. In particular, the synaptic connection between the modulatory CGCs and the CPG interneuron, N1M, was examined. In conditioned animals compared to controls, a significant increase in the size of inhibitory postsynaptic potentials (IPSPs) recorded in the N1M was observed following an artificial depolarization of the CGCs. Because the CGCs are known to play a critical gating role in feeding behavior (Yeoman et al. 1994) and the N1M is a pivotal member of the feeding CPG (Kemenes and Elliott 1994), this enhanced IPSP may be an important cellular correlate of the conditioned taste-aversion learning (Kojima et al. 1997).

\section{Operant Conditioning of the Respiratory Network}

The aerial respiratory behavior of $L$. stagnalis has been used in a series of experiments investigating the behavioral and cellular mechanisms of operant conditioning (Lukowiak et al. 1996, 1998; Spencer et al. 1999). Hypoxia triggers pneumostome opening and this was used as the operantly conditioned behavior. Tactile stimulation of the pneumostome area evokes pneumostome closure and stops aerial respiratory behavior. Animals were tested and trained in an artificially-created hypoxic nitrogen-rich environment to in-

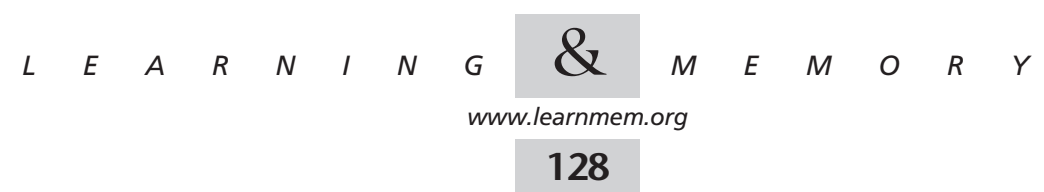


crease the level of respiratory behavior (Lukowiak et al. 1996). In the operantly trained group a tactile stimulus was applied to the pneumostome area each time aerial respiration was attempted by the animal. Suitable yoked and hypoxic control groups were also used. The number of openings, latency to first opening, and total breath durations were recorded in pre- and post-training periods. Only the operantly conditioned group showed significant changes between the pre- and post-training behaviors, with significant reductions in openings and total breathing time and significant increases in the latency to first breath (Lukowiak et al. 1996). It has since been demonstrated that a memory for this conditioned response could persist for at least 4 weeks when a spaced training procedure was used (Lukowiak et al. 1998).

More recently, neural changes associated with this learned behavior have been identified in the isolated CNS derived from operantly conditioned animals (Spencer et al. 1999). Specifically, spontaneous patterned activity in the IP3 interneuron, which is involved in pneumostome opening (see Fig. 1B) showed a significant reduction compared with activity in the IP3 neurons of brains derived from yoked controls. Furthermore, a higher percentage of RPeD1 CPG interneurons, which are important in the onset of the respiratory cycle, were silent in conditioned versus control preparations (Spencer et al. 1999). They also observed a reduction in the ability of the RPeD1 cells to induce IP3 activity. One speculation arising from these findings is that higher-order neurons involved in withdrawal responses, such as RPeD11, may be providing maintained inhibitory inputs to the respiratory CPG as a result of the operant conditioning (Spencer et al. 1999).

\section{Conclusions and Discussion}

The picture emerging from the work we have reviewed suggests that different forms of associative conditioning in Lymnaea result in electrical changes that can be recorded at several different locations in the nervous system. This represents an intermediate or systems level of analysis between behavior and the cellular and molecular studies of synaptic plasticity. It provides an opportunity to carry out a direct quantitative comparison of behavior and its electrophysiological correlate. The expression of the memory or its read-out (Byrne 1985) was mainly recorded as a global pattern of activity at the level of motoneurons and interneurons of the feeding and respiratory networks.

Our detailed knowledge of the Lymnaea feeding circuitry allowed us to infer that the rhythmic activity of the feeding CPG in response to the CS was increased following appetitive classical conditioning (Whelan and McCrohan 1996; Staras et al. 1998b, 1999a). Other changes associated with appetitive classical conditioning were recorded at the level of the motoneurons (enhancement of an early EPSP response) and the modulatory interneurons (activation of the CV1s). The activation of the synaptic connection between the CV1 modulatory interneuron and the CPG neurons is considered to be particularly important because CV1 cells can drive activity in the CPG network and this is likely to be essential to obtain a full readout of the feeding motor program. These results are consistent with a multistage or multisite model of appetitive classical conditioning but do not rule out a single common site of plasticity, particularly as a very short latency change in activity could be recorded in what was interpreted as part of the mechanosensory CS pathway. Recently, a pairing-specific strengthening of the mechanoafferent pathway also has been found after tactile appetitive classical conditioning of feeding in Aplysia (Lechner et al. 2000). Changes at the level of sensory neurons have also been shown to be major sites of plasticity in aversive classical conditioning of both the gill withdrawal reflex of Aplysia (Hawkins et al. 1983) and phototactic behavior in Hermissenda (Crow and Alkon 1980).

The causal link between the various sites of electrical change after appetitive classical conditioning have not been established, but we can make certain inferences based on the temporal sequences of events recorded after the application of the CS following conditioning, and relating this information to our knowledge of the synaptic connectivity of the circuit (see Fig. 1A). These inferences led to a generalized working model for appetitive learning summarized in Figure 4. We know in naive animals (Fig. 4A) that sensory inputs, such as touch and amyl actetate, produce weak subthreshold synaptic responses in neurons of the feeding circuit (Staras et al. 1999b; G. Kemenes, unpubl.). Recent evidence indicates that distribution of the weak tactile inputs to the modulatory interneurons, the CPG interneurons and the motoneurons may occur via sensory integratory neurons (Fig. 4A) and we make the assumption here that the same is true for chemical inputs. Potential synaptic interactions between the three types of feeding target cells for sensory input are shown by the dashed lines in Figure 4A. After classical conditioning (Fig. 4B), the sensory inputs to the modulatory neurons (CV1), motoneurons (B3), and presumably the CPG cells, are facilitated. The facilitated sensory input makes the modulatory and CPG interneurons fire and the synaptic connections become operative (solid lines in Fig. 4B). The CPG neurons then drive rhythmic feeding activity in the motoneurons leading to sequences of muscular activity and feeding movements. Whether the specific plastic changes after tactile versus chemical conditioning are the same remains to be investigated.

Multiple changes were also recorded in the respiratory network after aversive operant conditioning of the aerial respiratory behavior (Spencer et al. 1999). This indicates that a multisite hypothesis of plastic changes after associative conditioning in Lymnaea is now strongly supported by work on both classical and operant conditioning. In the aversive classical conditioning paradigm used by Kojima et

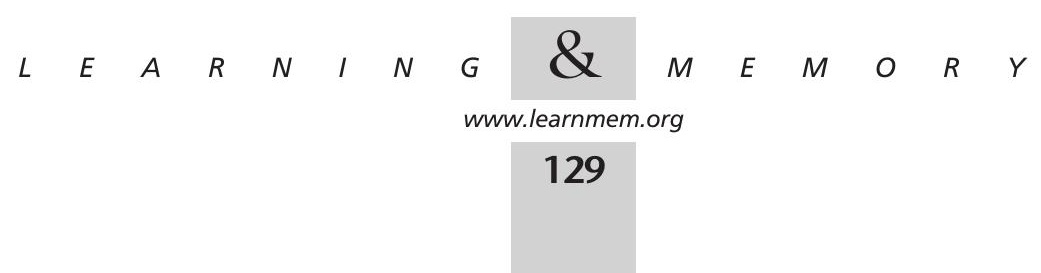



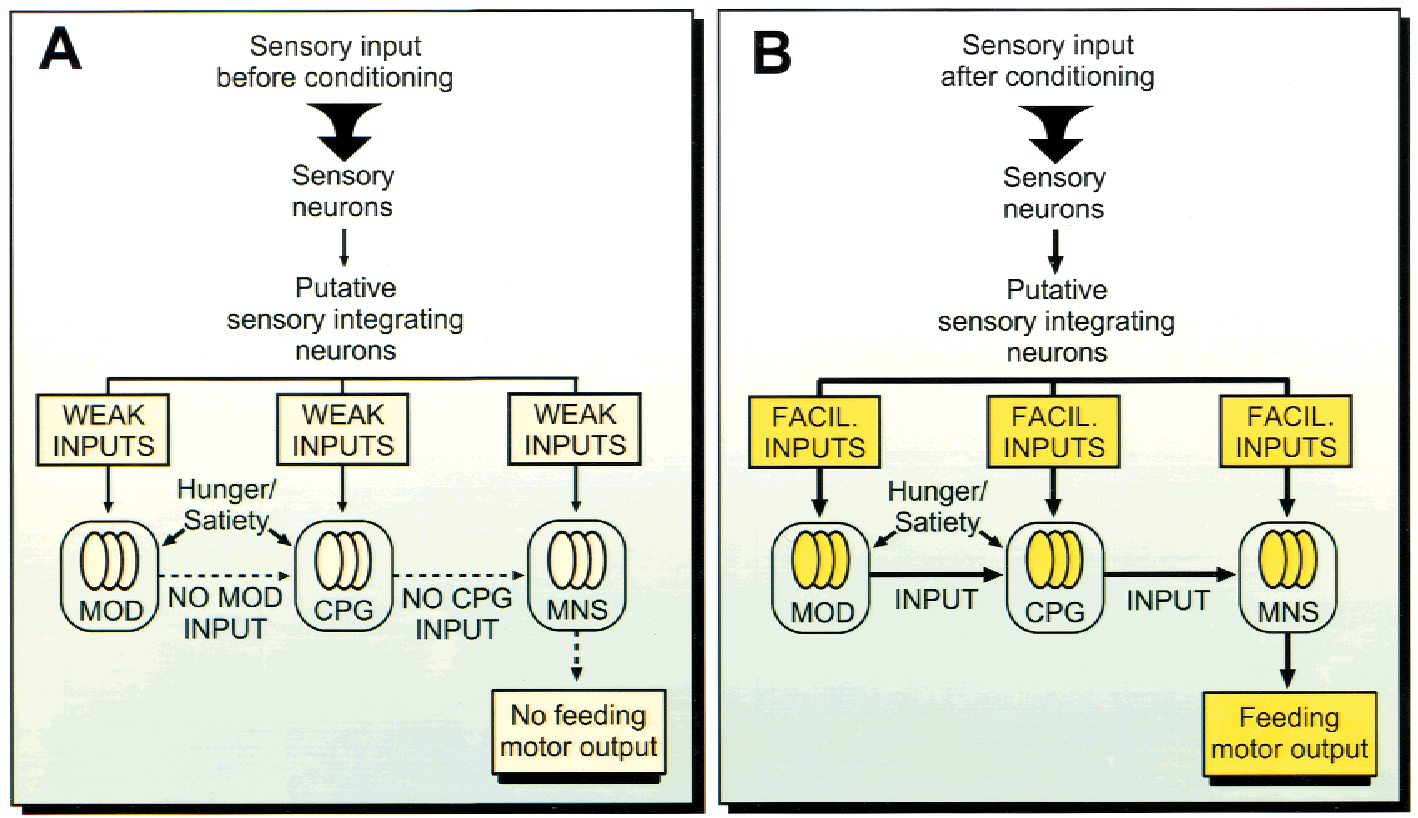

Figure 4 Summary of electrophysiological changes recorded after appetitive conditioning in Lymnaea. In naive animals $(A)$, a neutral sensory input causes weak synaptic responses as a result of parallel inputs to all members of the feeding circuit (thin lines). This may occur by way of putative sensory integratory neurons that distribute the weak sensory input to the modulatory interneurons (MOD), the CPG interneurons (CPG), and the motoneurons (MNS). Potential synaptic interactions between these three types of feeding target cells for sensory input (broken lines), are inoperative in naive snails as the effects of touch on the target cells are subthreshold for spike initiation. After conditioning $(B)$, the parallel sensory inputs to the modulatory neurons, the motoneurons, and presumably the CPG cells are facilitated (thick lines). The facilitated sensory inputs make the modulatory and CPG interneurons fire and the synaptic connections become operative (solid lines in B). Rhythmic feeding activity in the CPG neurons leads to patterned activity in the motoneurons and subsequently, feeding behavior.

al. (1997), only a single learning-related plastic change has been described, between the modulatory CGC and the CPG interneuron, N1M. However, this may not be a monosynaptic connection (Yeoman et al. 1996) and therefore a multisite hypothesis for this type of learning cannot be ruled out either.

The thorough understanding of the feeding and respiratory networks, both of which can undergo plastic changes during conditioning, establishes Lymnaea as a very valuable model organism for a variety of systems-level analyses of learning. In the future, our increasing knowledge of the synaptic connectivity and transmitter content of neurons in these networks (Elliott and Kemenes 1992; Yeoman et al. 1993, 1995, 1996; Santama et al. 1994; Inoue et al. 1996a,b; Brierley et al. 1997; Park et al. 1998; Perry et al. 1998; Staras et al. 1998a; Korneev et al. 1999; Magoski and Bulloch 1999) will allow a more detailed analysis of the molecular and cellular changes underlying associative conditioning.

\section{REFERENCES}

Alexander, J., T.E. Audesirk, and G.J. Audesirk. 1984. One-trial reward learning in the snail Lymnaea stagnalis. J. Neurobiol. 15: 67-72.

Audesirk, T.E., J.E. Alexander, G.J. Audesirk, and C.M. Moyer. 1982. Rapid, nonaversive conditioning in a freshwater gastropod. I. Effects of age and motivation. Behav. Neural Biol. 36: 379-390.
Benjamin, P.R. and C.J.H. Elliott. 1989. Snail feeding oscillator: The central pattern generator and its control by modulatory interneurons. In Neuronal and cellular oscillators (ed. J. Jacklet), pp. 173-214. Marcel Dekker, New York, NY.

Benjamin, P.R. and R.M. Rose. 1979. Central generation of bursting in the feeding system of the snail, Lymnaea stagnalis. J. Exp. Biol. 80: 93-118.

Benjamin, P.R. and W. Winlow. 1981. The distribution of three wide-acting inputs to identified neurons in the isolated brain of Lymnaea stagnalis. Comp. Biochem. Physiol. 70A: 293-307.

Brierley, M.J., M.S. Yeoman, and P.R. Benjamin. 1997. Glutamatergic N2v cells are central pattern generator interneurons of the Lymnaea feeding system: A new model for rhythm generation. J. Neurophysiol. 78: 3396-3407.

Byrne, J.H. 1985. Neural and molecular mechanisms underlying information-storage in Aplysia-implications for learning and memory. Trends Neurosci. 8: 478-482.

- 1987. Cellular analysis of associative learning. Phys. Rev. 67: 329-439.

Crow, T.J. and D.L. Alkon. 1980. Associative behavioral modification in Hermissenda: Cellular correlates. Science 209: 412-414.

Elliott, C.J.H. and P.R. Benjamin. 1985a. Interactions of pattern-generating interneurons controlling feeding in Lymnaea stagnalis. J. Neurophys. 54: 1396-1411.

. $1985 \mathrm{~b}$. Interactions of the slow oscillator interneuron with feeding pattern-generating interneurons in Lymnaea stagnalis. $J$. Neurophysiol. 54: 1412-1421.

Elliott, C.J.H. and G. Kemenes. 1992. Cholinergic interneurons in the feeding system of the pond snail Lymnaea stagnalis. II. N1 interneurons make cholinergic synapses with feeding motor neurons. Phil. Trans. R. Soc. Lond. B 336: 167-180.

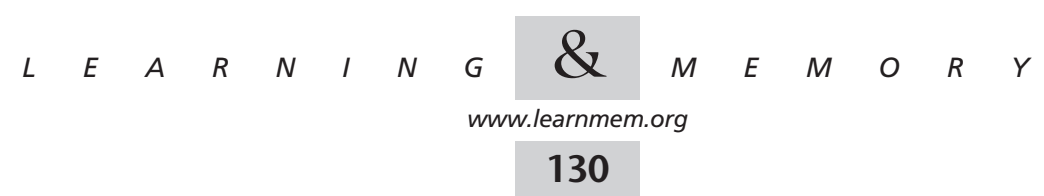


Fetcho, J.F. and D.M. O'Malley. 1997. Imaging neuronal networks in behaving animals. Curr. Opin. Neurobiol. 7: 832-838.

Hammer, M. 1997. The neural basis of associative reward learning in honeybees. Trends Neurosci. 20: 245-252.

Hawkins, R.D., T.W. Abrams, T.J. Carew, and E.R. Kandel. 1983. A cellular mechanism of classical conditioning in Aplysia: Activity-dependent amplification of presynaptic facilitation. Science 219: 400-405.

Inoue, T., M. Takasaki, K. Lukowiak, and N. Syed. 1996a. Identification of a putative mechanosensory neuron in Lymnaea: Characterization of its synaptic and functional connections with the whole-body withdrawal interneuron. J. Neurophysiol. 76: 3230-3238.

- $1996 \mathrm{~b}$. Inhibition of the respiratory pattern-generating neurons by an identified whole-body withdrawal interneuron of Lymnaea stagnalis. J. Exp. Biol. 199: 1887-1898.

Kemenes, G. and P.R. Benjamin. 1989a. Appetitive learning in snails shows characteristics of conditioning in vertebrates. Brain Res. 489: $163-166$

- 1989b. Goal-tracking behaviour in the pond snail Lymnaea stagnalis. Behav. Neural Biol. 52: 260-270.

- 1994. Training in a novel environment improves the appetitive learning performance of the snail, Lymnaea stagnalis. Behav. Neural Biol. 61: 139-149.

Kemenes, G., C.J.H. Elliott, and P.R. Benjamin. 1986. Chemical and tactile inputs to the Lymnaea feeding system: Effects on behaviour and neural circuitry. J. Exp. Biol. 122: 113-137.

Kemenes, G., K. Staras, and P.R. Benjamin. 1997. In vitro appetitive classical conditioning of the feeding response in the pond snail Lymnaea stagnalis. J. Neurophysiol. 78: 2351-2362.

Kojima, S., M. Yamanaka, Y. Fujito, and E. Ito. 1996. Differential neuroethological effects of aversive and appetitive reinforcing stimuli on associative learning in Lymnaea stagnalis. Zool. Sci. 13: 803-812.

Kojima, S., H. Nanakamura, S. Nagayama, Y. Fujito, and E. Ito. 1997. Enhancement of an inhibitory input to the feeding central pattern generator in Lymnaea stagnalis during conditioned taste-aversion learning. Neurosci. Lett. 230: 179-182.

Korneev, S.A., J.H. Park, and M. O'Shea. 1999. Neuronal expression of neural nitric oxide synthase (nNOS) protein is suppressed by an antisense RNA transcribed from an NOS pseudogene. J. Neurosci. 19: 7711-7720.

Lechner, H., D.A. Baxter, and J.H. Byrne. 2000. Classical conditioning of feeding in Aplysia: II. Neurophysiological correlates. J. Neurosci. 20, in press.

Lisberger, S.G. 1998. Cerebellar LTD: A molecular mechanism of behavioral learning? Cell 92: 701-704.

Lukowiak, K., E. Ringseis, G. Spencer, W. Wildering, and N. Syed. 1996. Operant conditioning of aerial respiratory behaviour in Lymnaea stagnalis. J. Exp. Biol. 199: 683-691.

Lukowiak, K., R. Cotter, J. Westly, E. Ringseis, and G. Spencer. 1998. Long-term memory of an operantly conditioned respiratory behaviour pattern in Lymnaea stagnalis. J. Exp. Biol. 201: 877-882.

Magoski, N.S. and A.G. Bulloch. 1999. Dopamine activates two different receptors to produce variability in sign at an identified synapse. $J$. Neurophysiol. 81: 1330-1340.

McCrohan, C.R. 1984. Initiation of feeding motor output by an identified interneurone in the snail Lymnaea stagnalis. J. Exp. Biol. 113: 351-366.

McCrohan, C.R. and P.R. Benjamin. 1980. Synaptic relationships of the cerebral giant cells with motoneurones in the feeding system of Lymnaea stagnalis. J. Exp. Biol. 85: 169-186.

Milner, B., L.R. Squire, and E.R. Kandel. 1998. Cognitive neuroscience and the study of memory. Neuron 20: 445-468.

Park, J.H., V.A. Straub, and M. O'Shea. 1998. Anterograde signaling by nitric oxide: Characterization and in vitro reconstitution of an identified nitrergic synapse. J. Neurosci. 18: 5463-5476.

Perry, S.J., V.A. Straub, G. Kemenes, N. Santama, B.M. Worster, J.F. Burke, and P.R. Benjamin. 1998. Neural modulation of gut motility by myomodulin peptides and acetylcholine in the snail Lymnaea. J. Neurophysiol. 79: 2460-2474.
Rose, R.M. and P.R. Benjamin. 1979. The relationship of the central motor pattern to the feeding cycle of Lymnaea stagnalis. J. Exp. Biol. 80: $137-163$.

- 1981. Interneuronal control of feeding in Lymnaea stagnalis. II. The interneuronal mechanisms generating feeding cycles. J. Exp. Biol. 92: 203-228.

Santama, N., M. Brierley, J.F. Burke, and P.R. Benjamin. 1994. Neural network controlling feeding in Lymnaea stagnalis: Immunocytochemical localization of myomodulin, small cardioactive peptide, buccalin, and FMRFamide-related peptides. J. Comp. Neurol. 342: 352-365.

Spencer, G.E., N.I. Syed, and K. Lukowiak. 1999. Neural changes after operant conditioning of the aerial respiratory behavior in Lymnaea stagnalis. J. Neurosci. 19: 1836-1843.

Staras, K., G. Kemenes, and P.R. Benjamin. 1998a. Pattern-generating role for motoneurons in a rhythmically active neuronal network. $J$. Neurosci. 18: 3669-3688.

- 1998b. Neurophysiological correlates of unconditioned and conditioned feeding behavior in the pond snail Lymnaea stagnalis. J. Neurophysiol. 79: 3030-3040.

- 1999a. Cellular traces of behavioral classical conditioning can be recorded at several specific sites in a simple nervous system. $J$. Neurosci. 19: 347-357.

- 1999b. Electrophysiological and behavioral analysis of lip touch as a component of the food stimulus in the snail Lymnaea. J. Neurophysiol. 81: 1261-1273.

Svoboda, K., W. Denk, D. Kleinfeld, and D.W. Tank. 1997. In vivo dendritic calcium dynamics in neocortical pyramidal neurons. Nature 385: 161-165.

Syed, N.I., A.G. Bulloch, and K. Lukowiak. 1990. In vitro reconstruction of the respiratory central pattern generator of the mollusk Lymnaea. Science 250: 282-285.

Syed, N.I, D. Harrison, and W. Winlow. 1991. Respiratory behavior in the pond snail Lymnaea stagnalis. I. Behavioral analysis and the identification of motor neurons. J. Comp. Physiol. A 169: 541-555.

Syed, N.I. and W. Winlow. 1991a. Coordination of locomotor and cardiorespiratory networks of Lymnaea stagnalis by a pair of identified interneurones. J. Exp. Biol. 158: 37-62.

- 1991b. Respiratory behavior in the pond snail Lymnaea stagnalis. II. Neural elements of the central pattern generator (CPG). J. Comp. Physiol. A 169: 557-568.

Whelan, H.A. and C.R. McCrohan. 1996. Food-related conditioning and neuronal correlates in the freshwater snail Lymnaea stagnalis. J. Moll. Stud. 62: 483-494

Wolpaw, J.R. 1997. The complex structure of a simple memory. Trends Neurosci. 20: 588-594.

Yeoman, M.S., D.C. Parish, and P.R. Benjamin. 1993. A cholinergic modulatory interneuron in the feeding system of the snail, Lymnaea. J. Neurophysiol. 70: 37-50.

Yeoman, M.S., A.W. Pieneman, G.P. Ferguson, A. Ter Maat, and P.R. Benjamin. 1994. Modulatory role for the serotonergic cerebral giant cells in the feeding system of the snail, Lymnaea. I. Fine wire recording in the intact animal and pharmacology. J. Neurophysiolol. 72: 1357-1371.

Yeoman, M.S., Á. Vehovszky, G. Kemenes, C.J.H. Elliott, and P.R. Benjamin. 1995. Novel interneuron having hybrid modulatory-central pattern generator-properties in the feeding system of the snail, Lymnaea stagnalis. J. Neurophysiol. 73: 112-124.

Yeoman, M.S., M.J. Brierley, and P.R. Benjamin. 1996. Central pattern generator interneurons are targets for the modulatory serotonergic cerebral giant cells in the feeding system of Lymnaea. J. Neurophysiol. 75: 11-25.

Received January 19, 2000; accepted in revised form March 14, 2000.

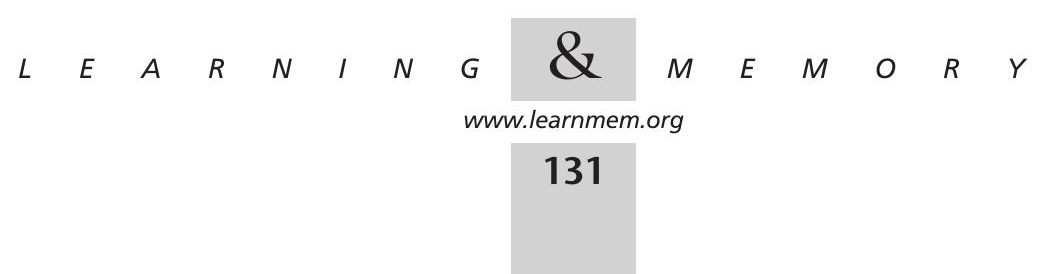




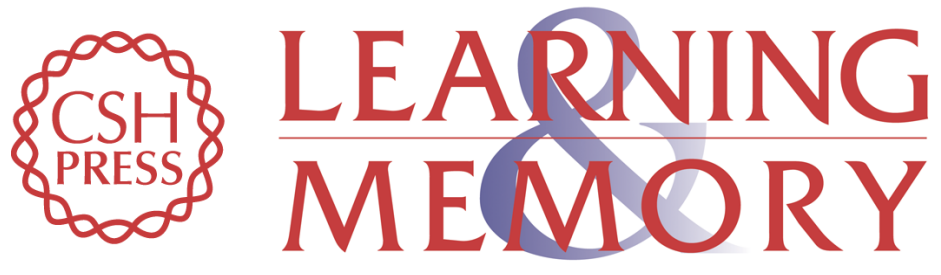

\section{A Systems Approach to the Cellular Analysis of Associative Learning in the Pond Snail Lymnaea}

Paul R. Benjamin, Kevin Staras and György Kemenes

Learn. Mem. 2000, 7:

Access the most recent version at doi:10.1101//m.7.3.124

References This article cites 51 articles, 18 of which can be accessed free at: http://learnmem.cshlp.org/content/7/3/124.full.htmI\#ref-list-1

License

Email Alerting Receive free email alerts when new articles cite this article - sign up in the box at the Service top right corner of the article or click here. 